\title{
Traveling Salesman Problem: A Case Study
}

\author{
Dr. Leena jain \\ Associate Professor \& Head-MCA \\ Global Institute of Management and \\ Emerging Technologies, \\ Amritsar, 143001 (India)
}

\author{
Mr. Amit Bhanot \\ Global Institute of Management \\ and Emerging Technologies, \\ Amritsar, 143001 (India)
}

\begin{abstract}
In this paper assignment based integer linear formulation presented for solving traveling salesman problem. Unfortunately, the assignment model can lead to infeasible solutions. Infeasibility removes by introducing additional constraints. Then this linear problem solved by open source software.
\end{abstract}

\section{General Terms}

Optimization technique, Linear Programming, Assignment problem. TSP, combinatorial optimization problem

\section{Keywords}

Optimization technique, Linear Programming, Assignment problem. TSP, combinatorial optimization problem

\section{INTRODUCTION}

The Traveling Salesman Problem (TSP) is a classical combinatorial optimization problem, which is simple to state but very difficult to solve. The problem is to find the shortest possible tour through a set of $\mathrm{N}$ vertices so that each vertex is visited exactly once. This problem is known to be NPcomplete, and cannot be solved exactly in polynomial time because the number of possible routes increases factorally with the number of cities. In other words, for 5 cities there are 5 ! (120) possible routes and for 6 cities there are 6! (720). A salesman who had to visit the 15 European capitals in this example would have $1,307,674,368,000$ (over 1.3 trillion) possible routes to consider. This traditional approach become impractical in terms of computer memory and speed constraints. So to solve this problem different researchers use heuristic, metaheuristic and optimal method like dynamic programming, linear programming. In this paper traveling salesman problem solved like assignment problem using linear programming approach. The constraints require that the salesman must enter and leave each city exactly once. Unfortunately, the assignment model can lead to infeasible solutions. Consequently, additional constraints must be included in order to eliminate subtour solutions. There are a number of ways to accomplish this.

\section{REVIEW OF LITERATURE}

The Traveling Salesman Problem (TSP) is one of the important subjects which have been widely addressed extensively by mathematicians and computer scientists [1]. Many approaches have been tried to solve TSP problem. Among them, there are polynomial-sized linear programming formulation [2], Genetic Algorithm [3,4,5,6], a mixed integer linear programming formulation and dynamic programming [7,8,9], NP-complete problem[10,11], Neural Network [12]. The multiple traveling salesman problem (mTSP) is a generalization of the well-known traveling salesman problem (TSP), where more than one salesman can be used in the solution [13].Historically, researchers have suggested a multitude of heuristic algorithms, including genetic algorithms (GA's) [14] for solving TSP [15]. Recently studied approaches to solve TSP includes Knowledge based multiple inversion and knowledge based neighborhood swapping GA algorithms [16], hybridized GA for integrated TSP and quadratic assignment problem [17], two-level GA for clustered TSP and large scale TSP [18], parallel GA program implementation on multicomputer cluster [19].

\section{ASSIGNMENT BASED \\ FORMULATION}

Starting from his home, a salesman wishes to visit each of ( $n$ $-1)$ other cities and return home at minimal cost. He must visit each city exactly once and it costs $c i j$ to travel from city $i$ to city $j$.

We may be tempted to formulate his problem as the assignment problem:

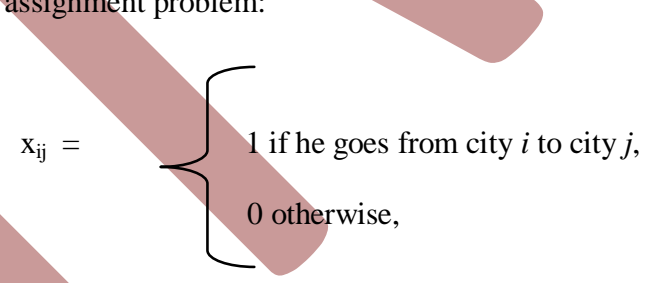

$\operatorname{Minimize} \sum_{i=1}^{n} \sum_{j=1}^{n} c_{i j} x_{i j}$

Subject to:

$$
\begin{aligned}
& \sum_{i=1}^{n} x_{i j}=1 \quad(j=1,2, \ldots, n), \\
& \sum_{j=1}^{n} x_{i j}=1 \quad(i=1,2, \ldots, n),
\end{aligned}
$$

Additional constraints

$x_{i j} \geq 0 \quad(i=1,2, \ldots, n ; j=1,2, \ldots, n)$.

The constraints require that the salesman must enter and leave each city exactly once. Unfortunately, the assignment model can lead to infeasible solutions. Infeasibility removes by additional constraints discussed in next section.

\section{EXPERIMENT SETUP}

To explain the procedure to solve traveling salesman problem we consider the following example in which we have five cities $1,2,3,4$ and 5 .

\begin{tabular}{l|lllll} 
From & 1 & 2 & 3 & 4 & 5 \\
\cline { 2 - 6 } 1 & - & 3 & 6 & 2 & 3
\end{tabular}




\begin{tabular}{l|lllll}
2 & 3 & - & 5 & 2 & 3 \\
3 & 6 & 5 & - & 6 & 4 \\
4 & 2 & 2 & 6 & - & 6 \\
5 & 3 & 3 & 4 & 6 & -
\end{tabular}

In the above problem we have 5 cities, let us designate the visit of $i^{\text {th }}$ city to $j^{\text {th }}$ city by the decision variable $x_{i j}$, $i=1,2,3,4,5$ and $j=1,2,3,4,5$ but $i \neq j$.

\section{Mathematical Formulation}
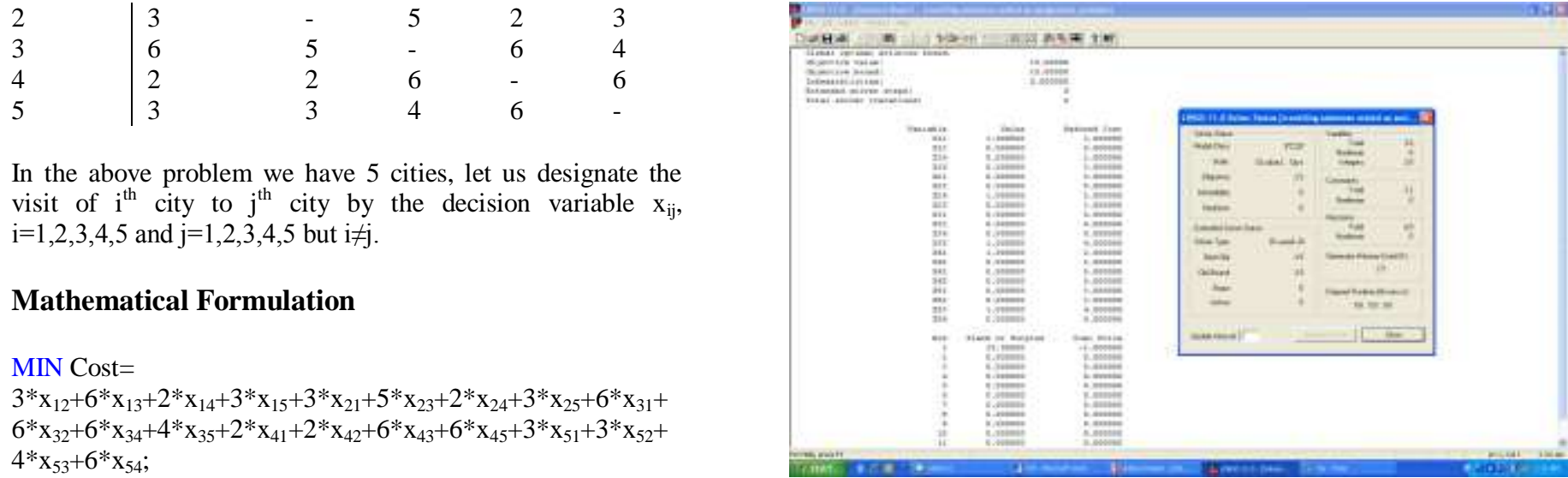

Subject to Constraints:

$\left(\mathrm{x}_{12}+\mathrm{x}_{13}+\mathrm{x}_{14}+\mathrm{x}_{15}\right)=1$

$\left(\mathrm{x}_{21}+\mathrm{x}_{23}+\mathrm{x}_{24}+\mathrm{x}_{25}\right)=1$

$\left(\mathrm{x}_{31}+\mathrm{x}_{32}+\mathrm{x}_{34}+\mathrm{x}_{35}\right)=1$

$\left(\mathrm{x}_{41}+\mathrm{x}_{42}+\mathrm{x}_{43}+\mathrm{x}_{45}\right)=1$

$\left(\mathrm{x}_{51}+\mathrm{x}_{52}+\mathrm{x}_{53}+\mathrm{x}_{54}\right)=1$

$\left(\mathrm{x}_{21}+\mathrm{x}_{31}+\mathrm{x}_{41}+\mathrm{x}_{51}\right)=1$

$\left(\mathrm{x}_{12}+\mathrm{x}_{32}+\mathrm{x}_{42}+\mathrm{x}_{52}\right)=1$

$\left(\mathrm{x}_{13}+\mathrm{x}_{23}+\mathrm{x}_{43}+\mathrm{x}_{53}\right)=1$

$\left(\mathrm{x}_{14}+\mathrm{x}_{24}+\mathrm{x}_{34}+\mathrm{x}_{54}\right)=1$

$\left(\mathrm{x}_{15}+\mathrm{x}_{25}+\mathrm{x}_{35}+\mathrm{x}_{45}\right)=1$

$\forall x_{i j} \geq 0(i=1,2, \ldots, 5 ; j=1,2, \ldots, 5)$.

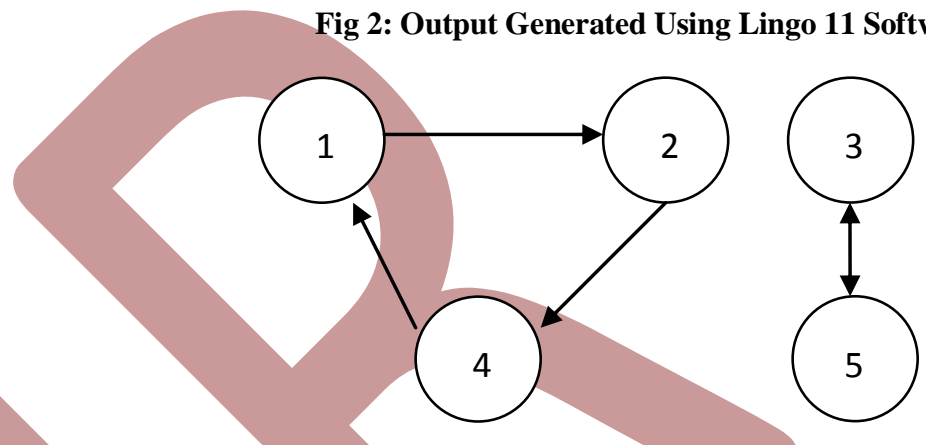

To solve the ILPP, Software LINGO Version 11 was used Figure 1 and Figure 2 illustrate the snap shots of construction of ILPP and generated solution by software LINGO 11 , respectively.

\section{Solution:}

$\mathrm{x}_{12}=\mathrm{x}_{24}=\mathrm{x}_{41}=\mathrm{x}_{35}=\mathrm{x}_{53}=1$

$\mathrm{x}_{13}=\mathrm{x}_{14}=\mathrm{x}_{15}=\mathrm{x}_{21}=\mathrm{x}_{23}=\mathrm{x}_{25}=\mathrm{x}_{31}=\mathrm{x}_{32}=\mathrm{x}_{34}=\mathrm{x}_{42}=\mathrm{x}_{43}=$

$\mathrm{x}_{45}=\mathrm{x}_{51}=\mathrm{x}_{52}=\mathrm{x}_{54}=0$

It is clear from the produced result assignment solution to route the salesman through disjoint subtours of the cities(1-24-1 and 3-5-3) instead of on a single trip or tour (figure 3)

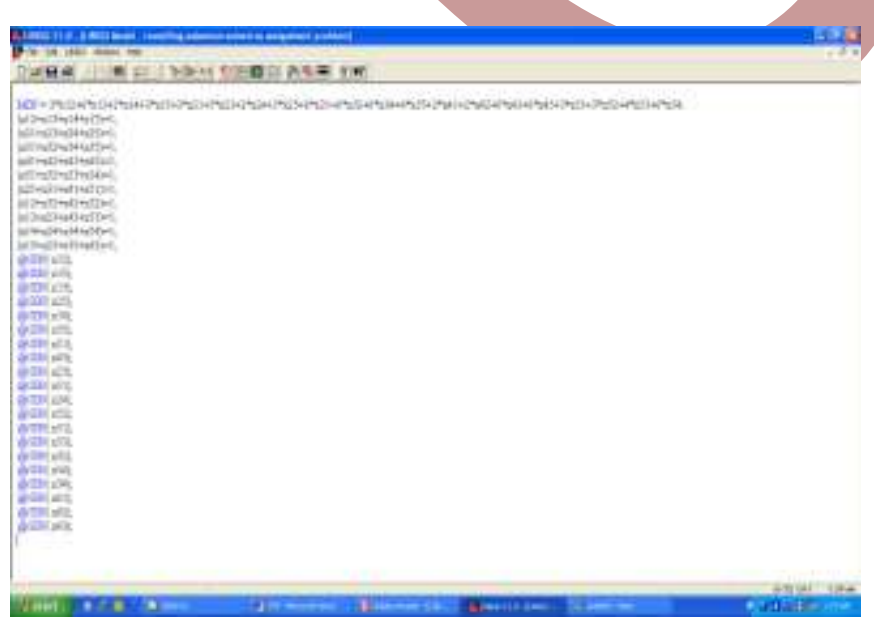

Fig 1: Formulation (Lingo 11 Software)

Unfortunately, the assignment model can lead to infeasible solutions. Infeasibility removes by additional constraints like $\mathrm{x}_{13}+\mathrm{x}_{15}+\mathrm{x}_{23}+\mathrm{x}_{25}+\mathrm{x}_{43}+\mathrm{x}_{45}>=1$; . ..(1)

This inequality ensures that at least one leg of the tour connects cities 1, 2, and 4 with cities 3 and 5 . In general, if a constraint of this form is included for each way in which the cities can be divided into two groups, then subtours will be eliminated. Now again above ILPP solved with additional constraint (1) using Software LINGO Version 11 that produce 1 tour cycle (fig 4).

\section{Solution:}

Minimum Cost $\mathrm{z}=16$

$\mathrm{x}_{14}=\mathrm{x}_{23}=\mathrm{x}_{35}=\mathrm{x}_{42}=\mathrm{x}_{51}=1$

$\mathrm{x}_{12}=\mathrm{x}_{13}=\mathrm{x}_{15}=\mathrm{x}_{21}=\mathrm{x}_{24}=\mathrm{x}_{25}=\mathrm{x}_{31}=\mathrm{x}_{32}=\mathrm{x}_{34}=\mathrm{x}_{41}=$

$\mathrm{x}_{43}=\mathrm{x}_{45}=\mathrm{x}_{52}=\mathrm{x}_{53}=\mathrm{x}_{54}=0$

Above result produce 1 tour cycle 1-4-2-3-5-1.

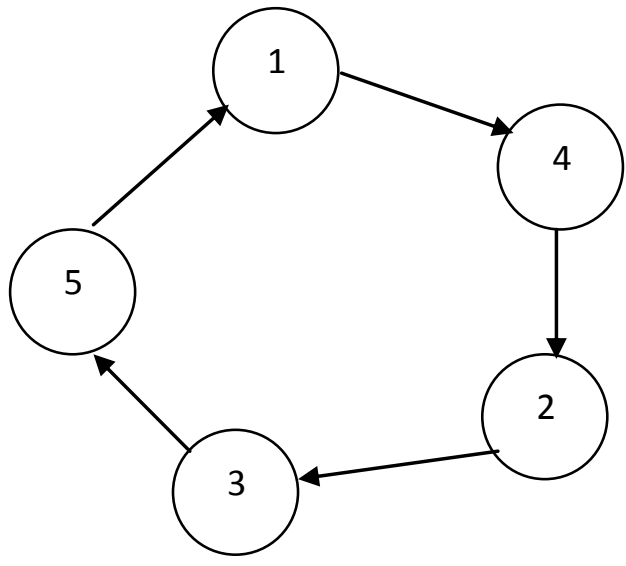

Fig 4: One Tour Cycle 


\section{CONCLUSION}

In this paper assignment based integer linear formulation presented for solving traveling salesman problem. Unfortunately, the assignment model can lead to infeasible solutions. Infeasibility remove by introducing additional constraints. Then this linear problem solved by open source software. Due to the constraint of length of the paper only one case have been reported here. However, more than ten problems have been solved using this approach.

\section{ACKNOWLEDMENT}

The authors are grateful to Mark Willey Lingo Systems, Inc., 1415 North Dayton, St. Chicago, IL 60622 for providing the complimentary copy of optimization software "Lingo11" for carrying out this study.

\section{REFERENCES}

[1] Buthainah Fahran Al-Dulaimi, and Hamza A. Ali," Enhanced Traveling Salesman Problem Solving by Genetic Algorithm Technique (TSPGA)", World Academy of Science, Engineering and Technology, Vol. 38, pp. 296-302, 2008.

[2] Moustapha Diaby, "The Traveling Salesman Problem: A Linear Programming Formulation", Wseas Transactions On Mathematics, Vol. 6, Issue 6. June 2007.

[3] Zakir H. Ahmed, "Genetic Algorithm for the Traveling Salesman Problem using Sequential Constructive Crossover Operator", International Journal of Biometrics \& Bioinformatics (IJBB) Vol.3, No.6, pp. 97-105.

[4] I. Choi, S. Kim and H. Kim. "A genetic algorithm with a mixed region search for the asymmetric traveling salesman problem". Computers \& Operations Research 30, pp. $773-786,2003$.

[5] C. Moon, J. Kim, G. Choi and Y. Seo. "An efficient genetic algorithm for the traveling salesman problem with precedence constraints". European Journal of Operational Research, Vol. 140, pp. 606- 617, 2002.

[6] D.E. Goldberg. "Genetic Algorithms in Search, Optimization, and Machine Learning". AddisonWesley, New York, 1989.

[7] C. Malandraki, M.S. Daskin, Time dependent vehicle routing problems: formulations, properties and heuristic algorithms, J. Trans. Sci., Vol. 26, pp. 185-200, 1992.

[8] C. Malandraki, R.B. Dial, A restricted dynamic programming heuristic algorithm for the time dependent traveling salesman problem, European J. Oper. Res., Vol. 90, pp. 45-55, 1996.
[9] R.J.V. Wiel, N.V. Sahinidis, Heuristic bounds and test problem generation for the time-dependent traveling salesman problem, J. Trans. Sci., Vol. 29, pp. 167-183, 1995.

[10] T. H. Cormen, C. E. Leiserson, R. L. Rivest, and C. Stein, Introduction to algorithms, (MIT Press, 2001).

[11] N. T. Shaked, S. Messika, S. Dolev, and J. Rosen, "Optical solution for bounded NP-complete problems," Appl. Opt. 46, pp. 711-724, 2007.

[12] S. Kedar, Naphade \& Dilek Tuzun, "Initializing the Hopfield-Tank Network for the TSP using a convex hull: A Computational Study. Proceedings of the Artificial Neural Networks in Engineering (ANNIE'95) Conference, pp. 399 - 404, 1995.

[13] Tolga Bektas, "The multiple traveling salesman problem: an overview of formulations and solution procedures", Omega The international journal of management science, Vol. 34, pp. 209-219, 2006 www.elsevier.com/locate/omega

[14] D. E. Goldberg, "Genetic Algorithm in Search, Optimization and Machine Learning", Machine Learning. Addison-Wesley, New York, 1989.

[15] P. Larranaga, C. M. H. Kuijpers, R. H. Murga, I. Inza and S. Dizdarevic, "Genetic Algorithms for the Travelling Salesman Problem: A Review of Representations and Operators", Artificial Intelligence Review. 13, pp. 129-170, 1999.

[16] S. R. Shubhra, B. Sanghamitra and K. Pal. Sankar, "New Operators of Genetic Algorithms for Traveling Salesman Problem", IEEE, 0-7695-2128-2/04, 2004.

[17] Ji. Ping and Ho. William, "The Traveling Salesman and the Quadratic Assignment Proble: Integration, Modeling and Genetic Algorithm", Int. Symposium on OR and its Applications, pp.198-205, 2005

[18] C. Ding, Ye. Cheng and M. He, "Two-Level Genetic Algorithm for Clustered Traveling Salesman Problem with Application in Large Scale TSPs", Tsinghua Science and Technology, Vol.12, No.4, pp. 459-465, 2007

[19] P. Borovska, T. Ivanova and H. Salem, "Efficient Parallel Computation of the Traveling Salesman Problem on Multicomputer Platform", Proceedings of the International Scientific Conference 'Computer Science' 2004, Sofia, Bulgaria, pp. 74-79, Dec. 2004. 